\title{
ABRUS PRECATORIUS TOXICITY PRESENTING WITH DIARRHOEA AND ENCEPHALOPATHY: A CASE REPORT
}

\author{
Shrestha $N,{ }^{1}$ Karki $B,{ }^{2}$ Regmi $S,{ }^{2}$ Shrestha PS, ${ }^{1}$ Acharya SP, ${ }^{1}$ Pathak $R^{1}$
}

\begin{abstract}
Infective etiologies are the first differentials that come to the mind in clinical conditions presenting with diarrhea associated with encephalopathy. We report a case of a 21 year old lady diagnosed as acute infective gastroenteritis and treated for the same only to manifest itself later as encephalopathy leading to a diagnostic dilemma. The clinical manifestations had started from abdominal cramps, bloody diarrhea followed by altered sensorium. A detailed history later revealed the use of the roots of Abrus precatorius for the treatment of sub-fertility was the missing piece of the diagnostic puzzle. Only after confirmation of the herb did the temporal sequence of events seemed logical. The patient was successfully managed for toxic acute disseminated encephalomyelitis (ADEM). Abrus precatorius is a commonly used herb used in traditional medicinal practice in many cultures. Various aspects of its toxicity can mimic other more commonly encountered medical ailments.
\end{abstract}

\section{KEYWORDS}

Abrus precatorious; acute disseminated encephalomyelitis; non-convulsive status epilepticus.

\section{CORRESPONDING AUTHOR}

Dr. Pramesh Sunder Shrestha,

Associate Professor

Department of Anaesthesiology,

Maharajgunj Medical Campus, Institute of Medicine,

Maharajgunj, Kathmandu, Nepal

Email: drpramesh@outlook.com

Orcid ID (1st Author): http://orcid.org/0000-0002-9416-596X

DOI: https://doi.org/10.3126/nmcj.v22i3.32657 


\section{INTRODUCTION}

Many infective organisms have been known to cause acute gastroenteritis associated with encephalopathy. ${ }^{1}$ Infective causes are the first differentials that come to the mind when faced with such a clinical scenario. However, different toxins can also have similar presentation which can cause a delay in the diagnosis and treatment. Anti-ribosomal toxins, abrin and ricin from the plant Abrus precatorius exhibit series of gastrointestinal disturbances like abdominal cramps, bloody diarrhea and vomiting. ${ }^{2}$ In rare cases, central nervous system (CNS) features of raised ICP, drowsiness, seizures, encephalitis and even death have been reported. ${ }^{3}$

Here we present a case of Abrus precatorius toxicity presenting with features of acute gastroenteritis followed by demyelinating encephalopathy which was successfully treated with steroids with no residual sequelae. Written informed consent was taken from the patient for the publication of this case report.

\section{CASE REPORT}

A 21 years lady with an alleged history of ingestion of some medicinal herb two hours back presented to a local health center with abdominal cramps, multiple episodes of vomiting and diarrhoea. She was managed symptomatically with intravenous fluids, antispasmodics and anti-emetics and discharged after a few hours of observation. After about six hours, the patient developed bloody diarrhea and was rushed back to hospital. The patient on investigation had a total white blood cell count of $46,000 /$ cumm. The patient was started on intravenous antibiotics and she was referred to our center the following day. During her four hours of journey in the ambulance, the patient had become drowsy and unresponsive to verbal commands. On arrival at the emergency department, her Glasgow coma scale (GCS) was E3V2M5 with stable vitals and maintaining an oxygen saturation of $98 \%$ in ambient air. Her investigations showed a leukocytosis of $31500 / \mathrm{cu} . \mathrm{mm}$ with neutrophilic predominance (83\%). The stool routine examination showed a red blood cell count of 8-10 cells per high power fields (HPF) and pus cells of 15-20/ hpf. She was transferred to the intensive care unit (ICU) in view of falling GCS. Point of care ultrasound on arrival at the ICU, revealed a raised optic nerve sheath diameter (ONSD) of $5.8 \mathrm{~mm}$ and $5.4 \mathrm{~mm}$ on the right and left respectively (Figure 1). On account of low GCS, a plain computerized tomography (CT) scan of the head was done which showed signs of raised ICP but was otherwise unremarkable. Intravenous mannitol was started and a lumbar puncture was obtained which revealed 5 cells (all lymphocytes) with a very high total protein content of $517 \mathrm{mg} / \mathrm{dl}$. An EEG was obtained which showed non-convulsive status epilepticus (NSCE) (Fig. 2). She was started on intravenous Levetiracetam. The diagnosis was then revised as acute gastroenteritis with encephalomyelitis. Injection ceftriaxone 2grams IV 12 hourly was started. The sample of the CSF was planned to be sent for herpes simplex virus (HSV), Japanese encephalitis (JE) and tubercular (TB) PCR.

In the meantime, presenting history was reviewed with the patient's husband in regards to the temporal sequence of events. The husband mentioned about the patient not being able to conceive for the last three years. The problem had been attributed to her irregular menses. A herbal medicine had been prescribed by a local healer to "cleanse the uterus" and the herb was said to be "(Rati Gedi)" which he further identified from a picture. It was further confirmed when he presented the actual herb.
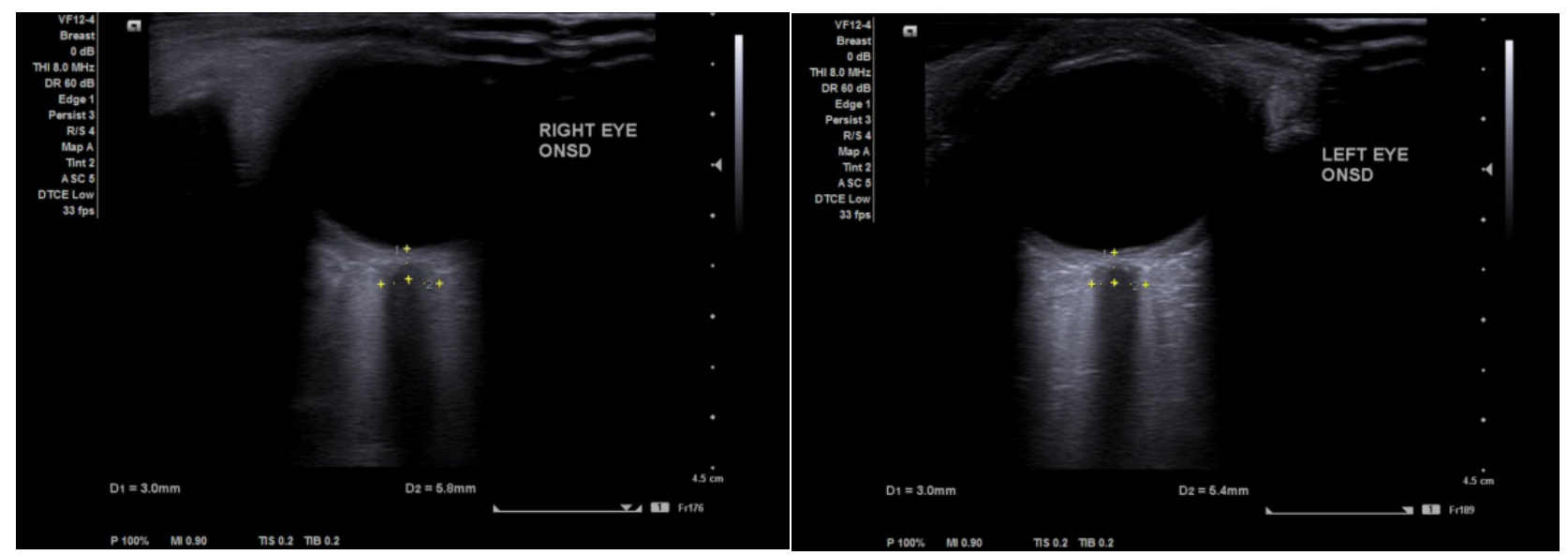

Fig. 1: Optic USG showing bilaterally raised ONSD 


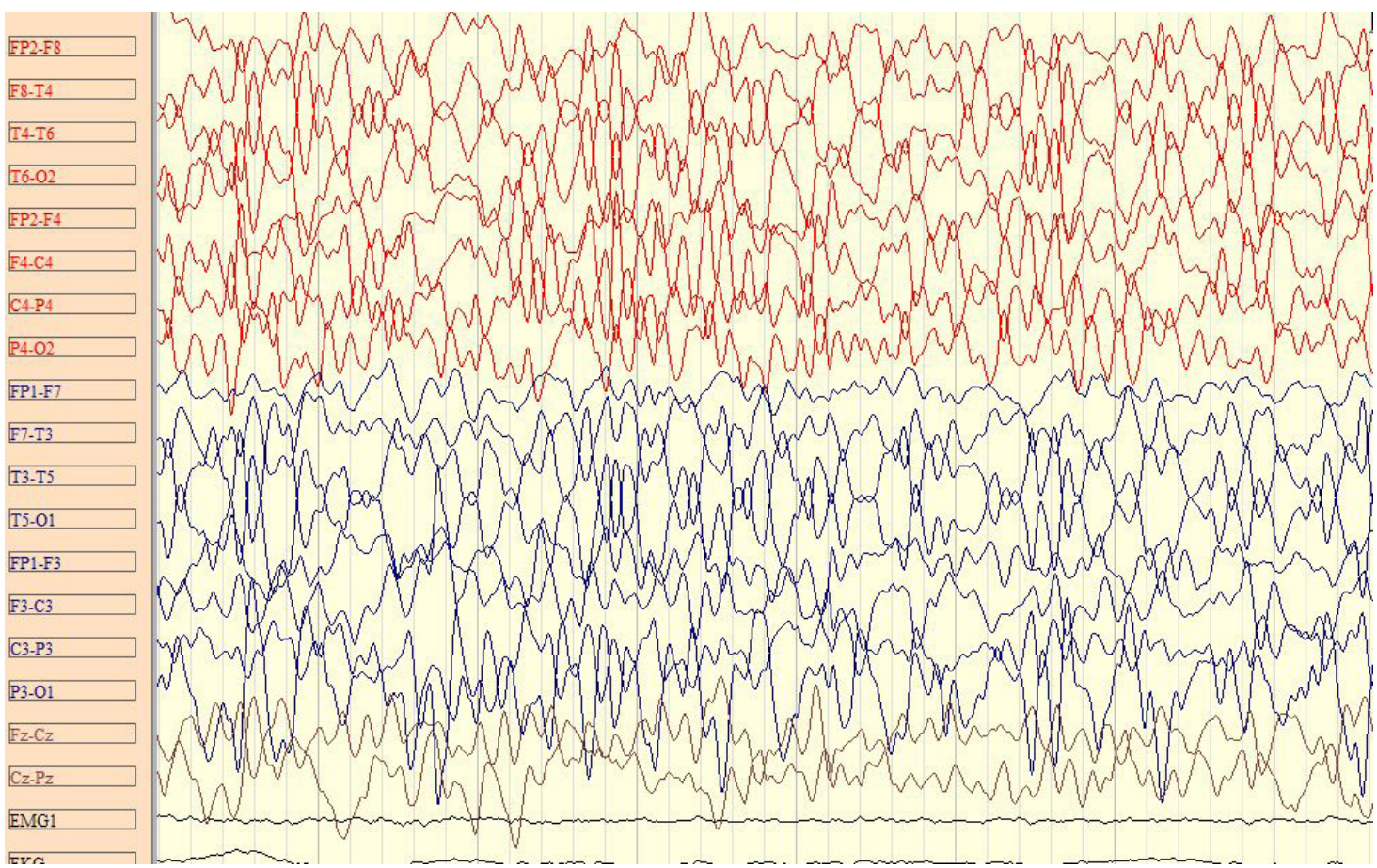

Fig. 2: EEG showing non-convulsive statue epilepticus
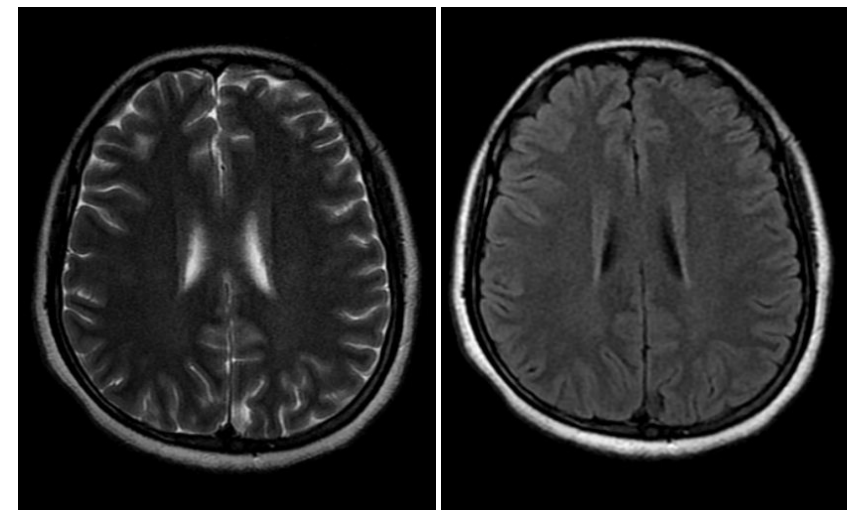

Fig. 3: MRI T2 and FLAIR Images showing diffuse demyelination

In light of the new evidence in the case, CSF microbial PCRs were withheld and a magnetic resonance imaging (MRI) was obtained which showed acute disseminated encephalomyelitis (ADEM) (Fig. 3). Antibiotic therapy was stopped and methyl-prednisolone was stared intravenously for three days which was later changed to oral prednisolone. On day 2 of the steroid therapy, the patient's EEG was repeated which still showed seizure activity and thus sodium valproate was added. By the evening of day 2 , the patient's seizure was controlled and the GCS had improved to E4V3M6. By day 3, the GCS was E4V4M6 and later $15 / 15$ by day 5 . The patient was transferred out from the ICU on day 7 and discharged home on day 10 with no neurological sequelae.

\section{DISCUSSION}

Various parts of the herb Abrus precatorius (rosary pea and called as Rati gedi locally) are frequently used as ayurvedic and home remedies for a myriad of ailments, especially the seeds and the roots. ${ }^{4}$ The plant has been shown to have very potent toxins abrin and ricin. They have multiple pharmacological, cytotoxic and immunomodulator effects. ${ }^{2,5}$ The most common uses of this herb are for eye problems like conjunctivitis, gastrointestinal problems, fever, and uterine problems including dysmenorrhoea,urticaria, eczema, stomatitis, alopecia areata, migraine, malaria,schistosomiasis, and lymphomas/ leukemia and is used even as a laxative, expectorant and aphrodisiac. ${ }^{4-6}$ The mechanism of action for the toxic effect has been found to be inactivation of the ribosome leading to de-purination of proteins and cytotoxicity. B subunit helps in attachment and entry of the A subunit inside the cell which stops the protein synthesis. ${ }^{2,7}$ The classical presentation of the poisoning following the herb ingestion has been reported to be abdominal cramps, vomiting followed by bloody diarrhea which has been found to be due to direct irritant, antiribosomal and cytotoxic effects. ${ }^{2}$

Severe leukocytosis have been reported which can lead to a diagnostic dilemma of an infective pathology. The CNS effects reported included rise in the ICP, demyelination and seizures. ${ }^{7}$ The ultrasonographic measurement of the 
ONSD and the brain imaging were consistent with features of raised intracranial pressure. CSF analysis showing the high protein content with cytoalbuminergic dissociation consistent with disseminated demyelination which was further confirmed with the MRI. The EEG showed nonconvulsive status epilepticus. All of these findings were coherent by reported CNS effects though they have been reported to be rare. However, all these clinical findings made sense when the identity of the actual herb was confirmed. Any unsuspecting clinician can be easily mislead to diagnosing the patient as having an infective pathology.
Abrus precatorius is a commonly used herb used in traditional medicinal practice in many cultures. Various aspects of its toxicity can mimic other more commonly encountered medical ailments. This report is an attempt to describe the toxicity profiles of this herb which is rarely encountered and very perplexing to an unsuspecting clinician. Any clinician should be aware of the other alternate faculties of medicine being practiced. Inability to timely suspect and diagnose such a condition may be deleterious to the patient's health.

\section{REFERENCES}

1. Ephros M, Cohen D, Yavzori M, Rotman N, Novic B, Ashkenazi S. Encephalopathy associated with enteroinvasive Escherichia coli 0144:NM infection. J Clin Microbiol 1996; 34: 2432-4.

2. Gadadhar S, Karande AA. Abrin Immunotoxin : Targeted Cytotoxicity and Intracellular Trafficking Pathway. PLoS One 2013; 8: 1-11.

3. Sahoo R, Hamide A, Amalnath SD, Narayana BS. Acute demyelinating encephalitis due to Abrus precatorius poisoning - complete recovery after steroid therapy. Clincal Toxicol 2008; 3650: 10713.

4. Acharya R, Acharya KP. Ethnobotanical study of medicinal plants used by Tharu community of Parroha VDC, Rupandehi district, Nepal. Sci World 2009; 7: 80-4.
5. Sheikh SG, Hedge K. Therapeutic Uses of Abrus precatorius: A Review. Indian J Pharma Chem Res 2017; 3: 196-201.

6. Ndamba J, Nyazema N, Makaza N, Anderson C, Kaondera K. Traditional herbal remedies used for the treatment of urinary schistosomiasis in Zimbabwe. J Ethnopharmacol 1994; 42: 125-32.

7. Subrahmanyan D, Dks JM, Raj M. An unusual manifestation of Abrus precatorius poisoning: A report of two cases. Clincal Toxicol 2008; 46: 173-5. 